SLEEP DISORDERED BREATHING

\title{
Effect of upper airway obstruction in acute stroke on functional outcome at 6 months
}

\author{
P M Turkington, V Allgar, J Bamford, P Wanklyn, M W Elliott
}

Thorax 2004;59:367-371. doi: 10.1136/thx.2003.005348

See end of article for authors' affiliations

.....................

Correspondence to: DrM W Elliott, Department of Respiratory Medicine, $\mathrm{St}$ James's University Hospital, Beckett Street, Leeds LS9 TTF, UK; mark. elliott@lineone.net

Received

13 February 2003

Accepted

7 November 2003
Background: The aim of this study was to determine whether upper airway obstruction occurring within the first 24 hours of stroke onset has an effect on outcome following stroke at 6 months. Traditional definitions used for obstructive sleep apnoea (OSA) are arbitrary and may not apply in the acute stroke setting, so a further aim of the study was to redefine respiratory events and to assess their impact on outcome.

Methods: 120 patients with acute stroke underwent a sleep study within 24 hours of onset to determine the severity of upper airway obstruction (respiratory disturbance index, RDI-total study). Stroke severity (Scandinavian Stroke Scale, SSS) and disability (Barthel score) were also recorded. Each patient was subsequently followed up at 6 months to determine morbidity and mortality.

Results: Death was independently associated with SSS (OR (95\% CI) 0.92 (0.88 to 0.95), $p<0.00001$ ) and RDI-total study (OR (95\% Cl) 1.07 (1.03 to 1.12), $\mathrm{p}<0.01)$. The Barthel index was independently predicted by SSS ( $p=0.0001 ; r=0.259 ; 95 \% \mathrm{Cl} 0.191$ to 0.327 ) and minimum oxygen saturation during the night ( $p=0.037 ; r=0.16 ; 95 \% \mathrm{Cl} 0.006$ to 0.184$)$. The mean length of the respiratory event most significantly associated with death at 6 months was 15 seconds (sensitivity 0.625 , specificity 0.525 ) using ROC curve analysis.

Conclusion: The severity of upper airway obstruction appears to be associated with a worse functional outcome following stroke, increasing the likelihood of death and dependency. Longer respiratory events appear to have a greater effect. These data suggest that long term outcome might be improved by reducing upper airway obstruction in acute stroke.
S troke disease is the third leading cause of death in the UK, after heart disease and cancer; an estimated $12 \%$ of all deaths are due to stroke. It accounts for an estimated $6 \%$ of total hospital costs in the UK, but the long term costs represent a huge financial burden on society. ${ }^{1}$ Interest has recently grown in the occurrence of sleep disordered breathing (SDB) and, in particular, obstructive sleep apnoea (OSA) following stroke. Two studies have estimated that $62 \%$ of stroke patients exhibit SDB, predominantly upper airway obstruction (UAO), in the first 24 hours after the onset of neurological symptoms. ${ }^{2}{ }^{3}$ In the immediate post stroke period areas of the brain are thought to become critically ischaemic, with areas in boundary zones and those supplied by terminal arteries most susceptible (the so called ischaemic penumbra), so any fluctuation in cerebral blood flow or blood oxygen saturation at this time may be critical. Previous studies have reported that up to $43 \%$ of stroke patients will have a progression of their neurological deficit. ${ }^{45}$ This typically occurs early after stroke onset, with $87 \%$ occurring within the first 48 hours, ${ }^{6}$ and has recently been shown to be independently associated with SDB. ${ }^{3}$ Blood pressure variability in patients with acute stroke has been shown to have an adverse affect on prognosis at 30 days. $^{7}$ This is probably because cerebral autoregulation is diminished after a stroke, ${ }^{8}$ thereby rendering cerebral perfusion pressure dependent. Haemodynamic oscillations in blood pressure, cardiac output, and heart rate ${ }^{9-12}$ are known to be associated with occlusion of the upper airway. Repeated episodes of UAO may therefore affect cerebral blood flow ${ }^{13-15}$ and cause fluctuations in intracranial pressure, ${ }^{15}{ }^{16}$ leading to further damage to the ischaemic penumbra. This may be further compounded by oxygen desaturation. Work by Balfors and Franklin ${ }^{17}$ has shown that, at the termination of an obstructive apnoea, blood pressure, cerebral blood flow and oxygen saturation are all reduced, thereby maximising the ischaemic potential of each episode of UAO. The main aim of this study was to determine whether UAO occurring in the first 24 hours after a stroke has a detrimental effect on functional outcome and prognosis.

Currently it is standard practice to define an apnoea as a 10 second cessation in airflow and a hypopnoea as a $50 \%$ reduction in airflow for 10 seconds associated with an oxygen desaturation or an arousal. This then allows the calculation of the respiratory disturbance index (RDI) as the number of apnoeas and hypopnoeas per hour of the study. A significant oxygen desaturation is usually defined as a fall from baseline of more than $4 \%$. These definitions, while convenient, are arbitrary and were designed to assess the impact of OSA on daytime function and, in particular, the maintenance of wakefulness. This may not be relevant in acute stroke as, for example, shorter apnoeas may still be associated with haemodynamic oscillations and therefore have an impact on critically ischaemic brain tissue. A secondary aim of this study was therefore to attempt to examine the length of apnoeas and hypopnoeas most associated with a poor functional outcome and to determine whether prolonged periods of UAO or short bursts of severe UAO have the greatest impact on outcome.

\section{METHODS}

\section{Subjects}

One hundred and twenty patients admitted to one of three wards in the Leeds Teaching Hospitals NHS trust who had suffered a stroke in the previous 24 hours were invited to participate. Although stroke patients were admitted to other

Abbreviations: CPAP, continuous positive airway pressure; GCS, Glasgow Coma Scale; OSA, obstructive sleep apnoea; RDI, respiratory disturbance index; SDB, sleep disordered breathing; SSS, Scandinavian Stroke Scale; UAO, upper airway obstruction 
wards during the recruitment period, they were excluded because stroke admissions to these wards were not frequent enough to ensure that nursing staff maintained their familiarity with the monitoring equipment used. Written consent was obtained from either the patient or their next of kin. Consent was sought as soon as possible after admission to the ward and the studies started directly thereafter. All patients admitted to the Leeds Teaching Hospitals NHS trust with stroke are registered on a database. We were therefore able to determine whether the sample studied was representative. The study was approved by the local ethics committee.

\section{Baseline and follow up assessments}

Strokes were classified into the four Oxford Community Stroke Project (OCSP) clinical subtypes. ${ }^{18}$ Stroke severity was graded using the Scandinavian Stroke Scale (SSS). ${ }^{19}$ The Glasgow Coma Scale (GCS) ${ }^{20}$ and limb weakness (Motricity index ${ }^{21}$ were documented on admission, and disability was assessed using the Barthel index. ${ }^{22}$ A CT brain scan was performed in each patient in the first 72 hours to confirm the diagnosis and pathological type of stroke. Previous history of stroke was documented (if patients or their relatives recalled a prior event or a previous admission with stroke had been documented in the medical notes). The patients were followed up at 6 months by a researcher blinded to their initial sleep study to prevent observer bias. The length of hospital admission (defined as date of admission to date of discharge to permanent place of residence so any time spent in a rehabilitation ward or hospital was included as part of their stay) was recorded. A standard questionnaire was sent to each patient to be completed either by them or by their carer. If the questionnaire was not returned by 14 days a further questionnaire and letter were sent. If the questionnaire was still not returned 14 days later a telephone call was made to their place of residence and, if possible, the questionnaire completed over the telephone. Data from the questionnaire were used to calculate residual disability (Barthel index), place of residence (nursing home, residential home or own home), whether the patients described themselves as independent or dependent on other people's help to carry out their daily tasks (dependency), and death.

\section{Sleep studies}

Studies were carried out using the Alice 4 sleep system (Respironics, Paris, France) and were started as soon as possible after admission and continued up to a total of 24 hours or until patients requested that the equipment be removed (each patient had at least 6 hours of data recorded during the night). Oronasal airflow (thermistor), heart rate (electrocardiogram), oxygen saturation (finger probe), abdominal and respiratory effort (strain gauge), snoring (microphone), body position (sensor detecting eight points of compass on thoracic strain gauge), and light intensity (light meter) were recorded. Each patient was studied in his or her own hospital bed and was not transported to the sleep laboratory. Usual nursing and physiotherapy practices were not altered during the study; in particular, the patient was positioned according to the usual ward protocol. Each study was individually scored by the same physician (PT) using standard criteria. An apnoea was defined as a 10 second cessation in airflow and a hypopnoea as a 50\% reduction in airflow for 10 seconds associated with a $4 \%$ oxygen desaturation. Baseline oxygenation was defined as the oxygen saturation in the first minute of the study. Respiratory disturbance index was expressed in three different ways (1) per hour of study (RDI-total study), (2) during the night only (RDI-lights out), and (3) during the hour when UAO was at its most severe (RDI-worst hour).

\section{Analysis of data}

Statistical analysis of all data was performed with SPSS version 9.0 for Windows. Logistic and linear regression analyses were used to assess the factors independently associated with outcome. Several variables were entered into the equation including stroke characteristics, patient demographic data, and sleep study variables. This was then repeated for RDI-lights out and RDI-worst hour. ROC curves using mean length of apnoea were used to calculate the mean length of apnoea which best predicted the outcome following stroke.

\section{RESULTS}

Of the 120 patients who underwent the initial sleep study analysis (data from whom have already been published in a previous report ${ }^{2}$ and are summarised in table 1 ), full follow up data were obtained in all but six patients (two refused to fill in the questionnaire and four could not be traced, at least one of whom had moved out of the region). There were no significant differences in the age and sex of the study population and all of the patients admitted with stroke in Leeds during the study period. Frequent UAO was found in the study population (61\% had RDI-total study $>10$ events/ $\mathrm{h})$; analysis of the night time only and worst hour data increased this further $(81 \%$ had RDI-lights out $>10$ events/h and $92 \%$ had RDI-worst hour $>10$ events/h). The mean delay from estimated time of onset of symptoms of stroke until the start of the sleep study was 10 hours 50 minutes. (For patients who awoke with their stroke, estimated time of onset of symptoms was taken as 05.00 hours.) Initial sleep study data of the patients followed up at 6 months are shown in table 2. Most of the respiratory events were obstructive in nature; only $9 \%$ of patients had predominantly central apnoea. Baseline oxygen saturation (defined as mean oxygen saturation in the first 5 minutes of each study) was not significantly different in the patients who were alive (mean (SD) $94.4(2.1) \%$ ) and those who had died (mean (SD) 94.3 $(2.5) \%)$ at 6 months. Table 3 illustrates the results of the 6 month follow up data and a comparison between patients with an RDI $>10$ and those with an RDI $<10$. Patients with frequent UAO (RDI $>10)$ had a higher mortality rate and the survivors spent a longer time in hospital than those with RDI $<10$ (fig 1). Patients with an RDI $<10$ survived longer than those with an RDI $>10$.

Logistic regression analysis was used to predict mortality, dependency, and place of residence at 6 months. Linear regression was used to predict disability (Barthel index). Confounders such as stroke characteristics (SSS, subtype, GCS, limb weakness, and whether or not this was the first stroke), basic demographic data (age, body mass index, neck circumference, hypertension, diabetes), and oxygen saturation data (desaturation index, minimum oxygen saturation, and baseline oxygen saturation) were entered into the equation. Sleep study data were entered as a continuous

\begin{tabular}{ll}
$\begin{array}{l}\text { Table } 1 \\
\text { subjects }\end{array}$ & Basic demographic data of study \\
\hline & All patients \\
\hline Age (years) ${ }^{*}$ & $79.3(10.6)$ \\
Sex (\% male) & $41.7 \%$ \\
Glasgow Coma Scale† & $15(12-15)$ \\
Limb weakness (Motricity index)† & $104(43.5-151)$ \\
Barthel index on admissiont & $3(0-9)$ \\
First stroke (\%) & $73.3 \%$ \\
Hypertensive (\%) & $35.8 \%$ \\
Diabetic (\%) & $16.7 \%$ \\
\hline *Mean (SD). & \\
†Median (interquartile range). & \\
\hline
\end{tabular}




\begin{tabular}{|c|c|}
\hline & All patients \\
\hline $\begin{array}{l}\text { RDI-total study (events } / \mathrm{h} \text { ) } \\
\text { RDI-lights out (events } / \mathrm{h} \text { ) } \\
\text { RDI-worst hour (events/h) } \\
4 \% \text { DI (desaturations per hour) } \\
\text { Time saturation }<90 \% \text { (min) } \\
\text { Baseline oxygen saturation (\%) } \\
\text { Snores per hour }\end{array}$ & $\begin{array}{l}14.5(7-25.3) \\
29.7(13.5-40)^{*} \\
42.5(21.3-55.8)^{*} \\
33(10-70) \\
13.5(4-55) \\
94.3(2.2) \dagger \\
33(8.2-54)\end{array}$ \\
\hline \multicolumn{2}{|c|}{$\begin{array}{l}\text { All values are expressed as median (IQR) except tmean } \\
\text { (SD). } \\
\text { `Significant difference from RDI-total study assessed by } \\
\text { Mann-Whitney tests. } \\
\text { RDI-total study = respiratory disturbance index per hour of } \\
\text { study; RDI-lights out= respiratory disturbance index during } \\
\text { the night only; RDI-worst hour = respiratory disturbance } \\
\text { index during the hour when UAO was at its most severe; } \\
D I=\text { desaturation index per hour of study. }\end{array}$} \\
\hline
\end{tabular}

variable (RDI-total study). Table 4 shows the results of the logistic regression analysis. Stroke severity (SSS) and severity of UAO (RDI-total study) were independently associated with death (positive predictive value (PPV) 83.5\%, negative predictive value (NPV) 55.8\%, overall classification 73.3\%) and dependency (PPV 97.5\%, NPV 33.3\%, overall classification $80.4 \%$ ). Stroke severity (SSS), age, and limb weakness (Motricity index) were independently associated with living in a nursing home at 6 months. Stroke severity $(0.573,95 \%$ CI $(0.473$ to 0.629$), p=0.0001$ change per one point fall in the SSS) and the minimum saturation recorded during the study $(0.161,95 \%$ CI 0.08 to 0.185$), p=0.033$ change per $1 \%$ fall in oxygen saturation) were independently associated with disability (Barthel index) at 6 months (the residuals were normally distributed mean (SD) $0.0(0.99)$ ).

When the regression analysis was repeated using RDIlights out instead of RDI-total study the results remained the same, except the utility of the model improved for both death (PPV $85.2 \%$, NPV 66.7\% and overall classification $77.8 \%$ ) and dependency (PPV 97.8\%, NPV 50\% and overall classification $89.1 \%)$. When RDI-worst hour was used there was no further improvement in the model utility over and above RDI-lights out.

Analysis using a ROC curve for mean length of respiratory event (either apnoea or hypopnoea) in each study suggested that the length of respiratory event most significantly associated with death was 15 seconds (sensitivity 0.625, specificity 0.525 ).

\section{DISCUSSION}

The study has shown that UAO occurring within the first 24 hours after stroke is associated with a worse functional outcome, increasing the likelihood of death and dependency at 6 months. Furthermore, stroke patients with frequent

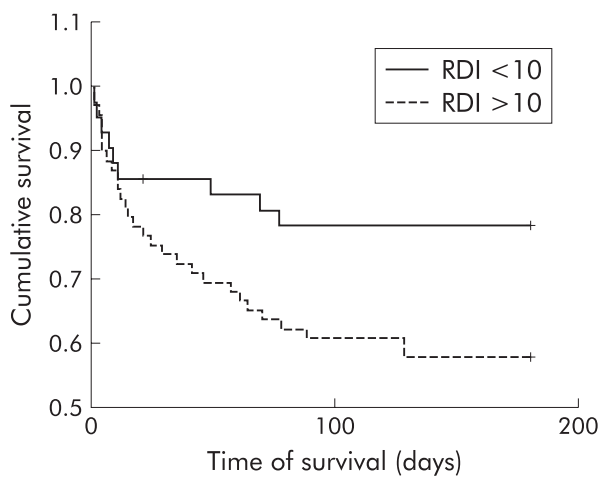

Figure 1 Kaplan-Meier survival plot. Stroke patients with a respiratory disturbance index (RDI) of $<10$ had significantly longer survival times than those with an RDI of $>10(p<0.04)$.

UAO appeared to die sooner than those without UAO, and the surviving patients spent longer in hospital if they experienced UAO. We have previously shown that there is no correlation between stroke severity and severity of $\mathrm{UAO}^{2}$ which would suggest that UAO is not just a marker of severe stroke. In addition, in this previous study other stroke characteristics (such as subtype and pharyngeal function), demographic factors (such as age and sex), and co-morbid medical conditions (such as hypertension and diabetes) also had no association with the presence of OSA following stroke.

The results would suggest that it is longer apnoeas and hypopnoeas that have the most deleterious effect. UAO occurring persistently throughout the night was a better predictor of outcome than short bursts of severe UAO.

The fact that UAO occurring within 24 hours of stroke affects the prognosis is not surprising, given the haemodynamic oscillations and fluctuations in oxygen saturation that accompany it. Blood pressure, pulse rate, cardiac output, and cerebral blood flow all decrease during obstructive apnoeas and hypopnoeas. ${ }^{9-12} 14$ At apnoea termination sudden increases in blood pressure, cardiac output, and pulse rate occur and are usually associated with a decrease in oxygen saturation. Neurological deterioration has been reported to occur in up to $43 \%$ of patients with stroke, ${ }^{45} 87 \%$ of which occurs within 48 hours. ${ }^{6}$ Iranzo et al have recently reported a link between this early neurological deterioration and SDB occurring within 24 hours of stroke onset, but were unable to demonstrate any association between the SDB and functional outcome 6 months after the stroke. ${ }^{3}$ However, their study differed from ours in several ways: their population was younger (mean (SD) age 66.8 (9.5) years), the severity of stroke appeared less (mean (SD) SSS 40 (13.5)), and they used full polysomnography. Two other studies have, however,

Table 3 Results of 6 month follow up

\begin{tabular}{lllll}
\hline & All patients & RDI $<10$ & RDI $>10$ & p valuet \\
\hline $\begin{array}{l}\text { Length of stay in hospital in survivors } \\
\text { (days)* }\end{array}$ & $25(10-64)$ & $24(10-39)$ & $43(15-71)$ & $<0.03$ \\
Stroke severity (SSS) & $30(16-42)$ & $30(16-43)$ & $30(18-41)$ & NS \\
Barthel index* & $1.5(0-13.5)$ & $1.5(0-14.25)$ & $2(0-11.75)$ & NS \\
Mortality (\% dead) & 36.7 & 25 & 45.2 & $<0.03$ \\
Dependency (\% "independent") & 16.7 & 18.1 & 16.3 & NS \\
Place of residence & 15.8 & 14.9 & 16.4 & NS \\
$\quad$ Nursing home (\%) & 29.2 & 32.9 & 23.4 & NS \\
Own home (\%) & & &
\end{tabular}

*Median (interquartile range, IQR).

†Significance tested by $\chi^{2}$ tests except those marked with an asterisk which are tested by Mann-Whitney $U$ tests. $\mathrm{RDI}=$ respiratory disturbance index; SSS = Scandinavian Stroke Scale. 
Table 4 Logistic regression analysis results

\begin{tabular}{|c|c|c|c|c|}
\hline Outcome & Significance & Odds ratio* & Lower $95 \% \mathrm{Cl}^{*}$ & Upper $95 \% \mathrm{Cl}^{*}$ \\
\hline \multicolumn{5}{|l|}{ Death } \\
\hline SSS & 0.00001 & 0.92 & 0.88 & 0.95 \\
\hline RDI-total study & 0.001 & 1.07 & 1.03 & 1.12 \\
\hline \multicolumn{5}{|l|}{ Dependency } \\
\hline SSS & 0.0001 & 0.9 & 0.86 & 0.95 \\
\hline RDI-total study & 0.009 & 1.06 & 1.01 & 1.11 \\
\hline \multicolumn{5}{|l|}{ Nursing home } \\
\hline SSS & 0.0001 & 0.9 & 0.88 & 0.94 \\
\hline Age & 0.01 & 1.06 & 1.01 & 1.11 \\
\hline Limb weakness & 0.04 & 1.02 & 1.001 & 1.03 \\
\hline \multicolumn{5}{|c|}{$\begin{array}{l}\text { Significant variables are shown after controlling for confounding variables such as stroke subtype, Glasgow Coma } \\
\text { Score (GCS), limb weakness, whether or not this was the first stroke, age, body mass index, neck circumference, } \\
\text { hypertension, and diabetes. } \\
\text { *OR for every } 1 \text { unit increase in variable (that is, estimated risk of death increases by } 1.07 \text { for every } 1 \text { unit increase } \\
\text { in RDI or decreases by } 0.92 \text { for every } 1 \text { unit increase in SSS). }\end{array}$} \\
\hline
\end{tabular}

suggested that there is an association between SDB and snoring following stroke and a worse functional outcome. However, neither assessed the first 24 hour period following the stroke. Good et $a l^{23}$ studied 47 patients with pulse oximetry a median 15 days following their stroke and found that a number of the oximetry variables (mean oxygen saturation, time spent less than $90 \%$ saturation, number of desaturations, and desaturation index) correlated with the Barthel index at discharge and improvement in the Barthel index from admission to discharge; this correlation persisted at 3 and 12 months. These four oximetry variables also correlated with the ability to return home after discharge. Mean oxygen saturation and time spent with less than $90 \%$ saturation correlated with ability to live at home at 3 months and death at 12 months. Spriggs et $a l^{24}$ reported that the 6 month survival rates for stroke patients were $79 \%$ for those who "never snored" compared with 52\% in those who "always snored" before the stroke. The early (within 7 days) and late (after 7 days) mortality rates in non-regular snorers (4\% and $18 \%$ ) were also significantly lower than in regular snorers (15\% and 30\%). Results from our study confirm that UAO is associated with both mortality and morbidity following stroke when it occurs in the immediate post stroke period. However, because of the possibility of unsuspected and unmeasured confounders inherent in any observational study, this association cannot be taken as confirming causation.

In order to obtain a representative group of stroke patients, the only exclusion criterion was not arriving on a "study ward" within 24 hours of stroke onset. This potentially could have introduced a bias due to significant co-morbidities (for example, chronic obstructive pulmonary disease or congestive cardiac failure) which could have reduced the baseline oxygen saturation and therefore magnified the depth of desaturation occurring with each respiratory event. As a hypopnoea required desaturation of at least $4 \%$, this may have increased the number of hypopnoeas recorded (and hence the RDI), leading to an overestimate of the significance of UAO on the functional outcome after the stroke. However, the fact that there was no significant difference in baseline oxygen saturation in stroke patients who were alive or dead at 6 months, and inclusion of baseline oxygen saturation in the regression analysis which did not prove to be significant, would suggest that any such effect was minimal.

The traditional definitions of apnoeas and hypopnoeas as at least 10 second events and oxygen desaturations as a decrease of at least $4 \%$ are arbitrary, as previously discussed. ROC curve analysis in this study suggested that a 15 second cut off for apnoeas or hypopnoeas would be better at predicting outcome than the traditional 10 second cut off. This is of particular importance when considering treatment of UAO in acute stroke, as it may be that preventing longer respiratory events ( $>15$ seconds) will be sufficient to improve outcome. It is well accepted that continuous positive airway pressure (CPAP) delivered non-invasively by either a nasal or face mask is the most efficient way of treating UAO in patients with OSA. ${ }^{25}$ However, concerns have been raised as to whether stroke patients will tolerate CPAP, ${ }^{23}{ }^{26}$ and results from two studies have shown CPAP compliance rates of $50-70 \%$ in highly selected stroke patients in the stable phase following their stroke. ${ }^{27} 28$ Levels of compliance were lower in those with worse disability and lower conscious levels, which suggests that compliance may be even lower in the acute phase. A more recent study ${ }^{29}$ which aimed to implement CPAP in the acute phase has reported even lower levels of compliance $(<50 \%$ when starting at day 4 after the stroke). Of the 20 patients who could not tolerate CPAP, 14 refused any further CPAP after a 30 minute acclimatisation period during the day and only four of those who did tolerate the CPAP titration night continued to use CPAP after discharge from hospital. CPAP therapy just aimed at preventing more severe UAO (possibly with lower and therefore better tolerated pressures) and hence larger swings in blood pressure, cerebral blood flow, and oxygen saturation rather than conventional CPAP aimed at abolishing all events may therefore be effective treatment and might be easier to implement in acute stroke.

The finding that persistent UAO throughout the night is the best predictor of outcome and that UAO occurring during the day has little additive effect is also interesting. Although this may have been biased by the fact that, in our study, $80 \%$ of patients were admitted in the evening and therefore their studies were started between 20.00 and 22.00 hours, suggesting that the night time UAO had more effect simply because it occurred earlier in the ischaemic penumbra. However, if this was a true effect, it would suggest that treatment should be targeted during the night and that using CPAP during daytime naps may not be essential.

This study addresses two important issues. It has confirmed that UAO_-which is known to be common within the first 24 hours of stroke and to be associated with haemodynamic oscillation and oxygen saturation fluctuation-is associated with a worse functional outcome following stroke at 6 months, both in terms of morbidity and mortality. It has also shown that it is longer respiratory events and deeper oxygen desaturations occurring particularly at night that have the greatest detrimental effect. Several key issues now remain and need further investigation. Treatment of UAO in the acute stroke setting should be considered with the aim of determining whether it can improve functional outcome. CPAP is the "gold standard" treatment of OSA, but consideration should be given to how it is delivered (either 
fixed pressure or autotitration) and at what pressure. It may be that elderly patients with acute stroke will find CPAP difficult to tolerate, but this study suggests that, even if it is not possible to eliminate all obstructive respiratory events, then at least preventing longer events-and hence more severe haemodynamic oscillations and oxygen desaturations-may be effective in improving outcome.

\section{Authors' affiliations}

P M Turkington, M W Elliott, Department of Respiratory Medicine, The Leeds Teaching Hospitals NHS Trust, St James's University Hospital, Leeds, UK

V Allgar, Department of Medical Statistics, The Leeds Teaching Hospitals NHS Trust, St. James's University Hospital, Leeds, UK

J Bamford, Department of Neurology, The Leeds Teaching Hospitals NHS Trust, St James's University Hospital, Leeds, UK

P Wanklyn, Department of Elderly Medicine, The Leeds Teaching Hospitals NHS Trust, St James's University Hospital, Leeds, UK

This project was funded by the Stroke Association.

Conflicts of interest: none.

\section{REFERENCES}

1 Bergman L, van der Meulen JH, Limburg M, et al. Costs of medical care after first-ever stroke in The Netherlands. Stroke 1995;26:1830-6.

2 Turkington PM, Bamford J, Wanklyn P, et al. Prevalence and predictors of upper airway obstruction in the first 24 hours after acute stroke. Stroke 2002;33:2037-42.

3 Iranzo A, Santamaria J, Berenguer J, et al. Prevalence and clinical importance of sleep apnea in the first night after cerebral infarction. Neurology 2002;58:911-6.

4 Britton M, Roden A. Progression of stroke after arrival at hospital. Stroke 1985; 16:629-32.

5 Davalos A, Cendra E, Teruel J, et al. Deteriorating ischemic stroke: risk factors and prognosis. Neurology 1990;40:1865-9.

6 Toni D, Fiorelli M, Gentile M, et al. Progressing neurological deficit secondary to acute ischemic stroke. A study on predictability, pathogenesis, and prognosis. Arch Neurol 1995:52:670-5.

7 Dawson SL, Manktelow BN, Robinson TG, et al. Which parameters of beat-tobeat blood pressure and variability best predict early outcome after acute ischemic stroke? Stroke 2000;31:463-8.

8 Fieschi C, Agnoli A, Battistini N, et al. Derangement of regional cerebral blood flow and of its regulatory mechanisms in acute cerebrovascular lesions. Neurology 1968;18:1166-79.
9 Loredo JS, Zeigler MG, Ancoli-Israel S, et al. Relationship of arousals from sleep to sympathetic nervous system activity and BP in obstructive sleep apnoea. Chest 1999;116:655-9.

10 Guilleminault C, Connolly S, Winkle R, et al. Cyclical variation of the heart rate in sleep apnoea syndrome. Mechanisms, and usefulness of $24 \mathrm{~h}$ electrocardiography as a screening technique. Lancet 1984;1:126-31.

11 Guilleminault C, Motta J, Mihm F, et al. Obstructive sleep apnoea and cardiac index. Chest 1986;89:331-4.

12 Guilleminault C, Connolly SJ, Winkle RA. Cardiac arrhythmia and conduction disturbances during sleep in 400 patients with sleep apnoea syndrome. Am J Cardiol 1983;82:490-4.

13 Fischer A, Chaudhary B, Taormina $M$, et al. Intracranial hemodynamics in sleep apnea. Chest 1992;102:1402-6.

14 Netzer N, Werner P, Jochums I, et al. Blood flow of the middle cerebral artery with sleep-disordered breathing. Stroke 1998;29:87-93.

15 Hayakawa T, Terashima M, Kayukawa Y, et al. Changes in cerebral oxygenation and haemodynamics during sleep apnoeas. Chest 1996; 109:916-21.

16 Jenum P, Borgesen S. Intracranial pressure and obstructive sleep apnea. Chest 1989;95:279-83.

17 Balfors EM, Franklin KA. Impairment of cerebral perfusion during obstructive sleep apneas. Am J Respir Crit Care Med 1994;150:1587-91.

18 Bamford J, Sandercock P, Dennis M, et al. Classification and natural history of clinically identifiable subtypes of cerebral infarction. Lancet 1991;337:1521-6.

19 Scandinavian Stroke Study Group. Multicenter trial of hemodilution in ischaemic stroke: background and study protocol. Stroke 1985;16:885-9.

20 Teasdale G, Jennett B. Assessment of coma and impaired consciousness. A practical scale. Lancet 1974;2:81-4.

21 Stojcevic N, Wilkinson P, Wolfe C. Outcome measurement in stroke patients. In: Wolfe C, Rudd T, Beach R, eds. Stroke services and research. London: The Stroke Association, 1996.

22 Mahoney FI, Barthel DW. Functional evaluation: the Barthel index. Maryland State Med J 1965; 14:61-65.

23 Good D, Henkle J, Gelber D, et al. Sleep disordered breathing and poor functional outcome after stroke. Stroke 1996;27:252-9.

24 Spriggs DA, French JM, Murdy JM, et al. Snoring increases the risk of stroke and adversely affects prognosis. Q J Med 1992;83:555-62.

25 Jenkinson C, Davies RJ, Mullins R, et al. Comparison of therapeutic and subtherapeutic nasal continuous positive airways pressure for obstructive sleep apnoea: a randomised prospective parallel trial. Lancet 1999;353:2100-5.

26 Parra 0 . Sleep-disordered breathing and stroke: is there a rationale for treatment? Eur Respir J 2001; 18:619-22.

27 Wessendorf TE, Wang YM, Thilmann AF, et al. Treatment of obstructive sleep apnoea with nasal continuous positive airway pressure in stroke. Eur Respir 2001;18:623-9.

28 Sandberg O, Franklin KA, Bucht G, et al. Nasal continuous positive airway pressure in stroke patients with sleep apnoea: a randomized treatment study. Eur Respir J 2001;18:630-4.

29 Hui DS, Choy DK, Wong LK, et al. Prevalence of sleep-disordered breathing and continuous positive airway pressure compliance: results in Chinese patients with first-ever ischemic stroke. Chest 2002;122:852-60. 